\title{
Control of energy dissipation in sliding low-dimensional materials
}

\author{
Antonio Cammarata $\circledast^{*}$ and Tomas Polcar \\ Department of Control Engineering, Faculty of Electrical Engineering, Czech Technical University in Prague, \\ Technicka 2, 16627 Prague 6, Czech Republic
}

(Received 28 April 2020; revised 16 July 2020; accepted 27 July 2020; published 7 August 2020)

\begin{abstract}
Frictional forces acting during the relative motion of nanosurfaces are the cause of energy loss and wear which limit an efficient assembly and yield of atomic-scale devices. In this research, we investigate the microscopic origin of the dissipative processes as a result of the frictional response, with the aim to control them in a subtle way. We recast the study of friction in terms of phonon modes of the system at the equilibrium, with no need to resort to dynamics simulations. As a case study, we here consider layer sliding in transition metal dichalcogenides thin films. We find that the population of specific atomic orbitals and the relative contribution of the atomic type to selected system vibrations are the crucial quantities which determine the frictional response in tribological conditions. A reduced amount of energy dissipation is found when the bond character is more ionic and the layer sliding is realized by a faster motion of the chalcogen atoms. The individuated relevant parameters governing the energy dissipation can be used as descriptors in high-throughput calculations or machine learning engines to screen databases of frictional materials. The presented framework is general and can be promptly extended to the design of tribological materials with targeted frictional response, irrespective of the chemistry and atomic topology.
\end{abstract}

DOI: 10.1103/PhysRevB.102.085409

\section{INTRODUCTION}

Manipulation and assembly of free-standing atomic layers into final devices, and their use in micro/ nanoelectromechanical systems (MEMS/NEMS, e.g., sensors and actuators) require a deep knowledge and control of their frictional response and related energy dissipation characteristics [1]. Moving parts during device fabrication and operation are subject to nonconservative forces active during the relative motion of the involved surfaces; those forces limit the output efficiency by producing heat, fatigue and wear up until compromising the correct construction or functioning of the device. Indeed, the comprehension of mechanisms governing friction at the nanoscale is a forefront challenge to save energy and increase the lifetime and sustainability of miniaturized devices, in addition to improving their performance [2]. To this aim, in the present work we investigate the atomic detail of the dissipative processes generated by frictional forces occurring during relative motions of few atomic layers.

Several classical and quantum mechanical approaches have already been developed to study friction at the atomic scale, by considering both ad hoc defined interatomic force formulations and parameter-free $a b$ initio descriptions [3-6]. The reliability of such approaches is a compromise between the accuracy of the system-dependent parametrizations and/or the width of the simulated time-window-this last being computationally expensive if $a b$ initio methods are used [7-13]. We here follow a different approach, with the goal being to ob-

\footnotetext{
*cammaant@fel.cvut.cz
}

tain information on frictional and dissipative properties by the only knowledge of the static properties of the system, without the need to perform long and costly dynamic simulations; furthermore, we use a system-independent framework which is applicable to any kind of chemistry and atom topology. The approach that we adopt is based on the phonon modes calculated on the stable geometry; indeed, recent works have already drawn attention to the role of phonons in determining the frictional properties of tribological systems $[14,15]$.

We already showed that any sequence of geometric configurations representing the layer sliding can be decomposed in terms of polarization vectors obtained by diagonalization of the dynamical matrix at any point of the reciprocal space $[16,17]$. This allowed us to recast the study of the frictional response in terms of sliding and dissipative phonon modes [18]. The sliding modes give rise to relative shifts of adjacent atomic layers. In terms of the classical picture, to each of these modes we can associate a harmonic restoring force $f \propto$ $\omega^{2}$, where $\omega$ is the mode frequency; by lowering the mode frequency, it is then possible to lower the restoring force and hence facilitate the layer sliding. By going beyond the local harmonic description [18] we observed that the layer sliding occurs as long as the energy contained in the sliding modes (i.e., the phonon population) is above a certain threshold characteristic of the material. The dissipative modes instead are all those modes which subtract energy from the sliding modes by means of phonon recombination processes. Such processes correspond to energy dissipation since they degrade the ordered motion (sliding) into disordered vibrations (heat); in this way, we can define the frictional forces as those forces corresponding to phonon scattering events reducing the population of the sliding modes. 
(a)

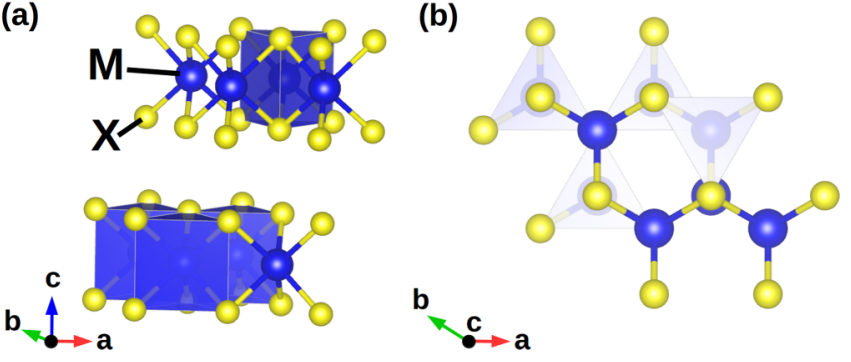

FIG. 1. (a),(b) Model structure of hexagonal $P 6_{3} / m m c 2 \mathrm{H}$ polymorph of $M X_{2}$ TMD crystal. $M-X$ bonds form trigonal prisms arranged in parallel layers which can reciprocally slide thanks to weak van der Waals interactions.

In the present work, we discuss which are the relevant quantities that determine such energy dissipation, and how to act to deactivate specific dissipation channels. We also show that this can be achieved by using quantum mechanical static calculations on the fixed geometry of the stable system, thus saving computational time and investigation effort which would be required by standard dynamical approaches. As a case study, we consider layer sliding in transition metal dichalcogenides (TMDs) thin films, which are becoming ubuquitous in several technological applications spanning hydrogen evolution, energy storage, high-performance electronics, and photonic devices [19-21].

\section{METHODS}

\section{A. Computational details}

Transition metal dichalcogenides have general stoichiometry $M X_{2}$, where $M$ and $X$ are a transition metal and a chalcogen atom, respectively. One $M$ cation coordinates six chalcogen anions in a trigonal prismatic arrangement forming a periodic $M X_{2}$ layer with $M-X$ covalent bonds (Fig. 1). Adjacent layers are bound together by weak van der Waals forces which allow relative sliding under tribological conditions. We consider the $2 \mathrm{H}$ polymorph crystalline $M X_{2}$ compounds as reference structures [19], with $M=\mathrm{Mo}, \mathrm{W}$ and $X=\mathrm{S}$, $\mathrm{Se}$, Te, and hexagonal $P 6_{3} / m m c$ symmetry (SG 194). The primitive unit cell of such geometry is formed by two adjacent layers arranged in such a way that a metal atom of one layer is aligned with two anions of the other one along the direction orthogonal to the layer planes ( $c$-axis in our setting-see Fig. 1). Starting from the chosen reference geometries, we truncate the periodic image repetition along the $c$ axis by setting the $c$ lattice parameter at $65 \AA$, and consider only two, three, four, five, and six $M X_{2}$ subsequent layers. In this way, for each considered compound, we build five model systems that we name $M X-n L$, where $M$ and $X$ specify the kind of transition metal and chalcogen atom, respectively, while $n$ corresponds to the number of $M X_{2}$ layers forming the unit cell; the corresponding space group is $P \overline{3} m 1$ (\#164) if $n$ is odd and $P \overline{6} m 2$ (\#187) if $n$ is even.

We perform density functional theory (DFT) calculations using the Perdew-Burke-Ernzerhof (PBE) energy functional [22] as implemented in VASP software [23,24]. We also take into account van der Waals interactions using the Grimme correction [25], which correctly reproduces the structural features, as we reported in previous works and references therein $[16,26,27]$. The Brillouin zone is sampled with a minimum of a $7 \times 7 \times 1 k$-point mesh and plane wave cutoff of $500 \mathrm{eV}$. Full structural (atoms and lattice) relaxations are initiated from diffraction data [28-33] and the forces minimized to a $0.5 \mathrm{meV}^{-1}$ tolerance. We diagonalize the dynamical matrices of the stable systems with the aid of the PHONOPY software [34] and compute the first-order anharmonic phonon-phonon interaction strengths by means of the PHONO3PY software [35].

\section{B. Theoretical background}

In tribological conditions, the relative motion among subsequent $M X_{2}$ layers can be geometrically described in terms of eigendisplacements associated to the phonon modes of the stable geometry $[16,17]$. If we consider a system with $N$ atoms, the degrees of freedom of the system is $3 N$, with each atom being free to move along all the three spatial directions. At any $\boldsymbol{q}$ vector of the Brillouin zone of the system, the diagonalization of the dynamical matrix yields $3 N$ eigenvectors, each characterized by a polarization vector describing the atomic displacement pattern (i.e., the phonon mode pattern). The $3 N$ eigenvectors are orthogonal and constitute a basis for the geometric description of the system; there is therefore a one-to-one correspondence between the degrees of freedom of a system and the eigendisplacements relative to any $\boldsymbol{q}$ reciprocal vector [36], and any set of atomic displacements can then be represented as a linear combination of phonon modes. The projection of the geometric sequence representing the layer sliding onto the phonon eigenvectors allows to identify which phonons have the main role in the representation of the sliding motion: they are those with the largest coefficient in the linear combination, and we can name them as sliding modes. This phonon-based description of the layer sliding is not only a convenient geometric picture, but also provides fundamental information about the role of the phonons during the whole tribological process. This is apparent in the frequency analysis of dynamical trajectories [18]: sliding modes are active during the sliding motion while become silent once the sliding terminates. Layer sliding is active as long as the sliding modes own enough energy: scattering processes reduce the population of the sliding modes until the ordered motion (i.e., sliding) is completely downgraded to thermal vibration (i.e., heat) and the equilibrium is reached. The decomposition of sliding trajectories into phonon modes allows one to distinguish then between sliding and dissipative modes: the former are those who have an effective geometric contribution to the layer drift, while the latter reduce the population of the sliding ones via phonon-phonon recombination processes. In this description, the frictional forces are then all those forces which activate recombination processes that reduce the population of the sliding modes. In general, the main effect of friction during sliding is to increase the temperature of the system, while others like free charge production or structural deformations are minor ones; in our phonon-based description, we neglect such minor effects and frictional forces have the only consequence to convert work (ordered motion of the atoms represented by 
the sliding) into thermal vibrations, hence heat which rises the temperature of the system.

It is worth noting that we do not need to make any assumption on the sliding direction since such information is automatically transferred into the eigenvector linear combination by the projection of the cartesian displacements onto the polarization vectors. Moreover, the roughness of interfaces and presence of defects can be straightforwardly included in the present approach without the need for specific working hypotheses, provided that such irregularities are represented by an appropriate choice of the atomic geometry and supercell size. However, our representation is valid if the set of phonon eigenvectors constitute a good description of the system in tribological conditions, that is, if the equilibrium atomic topology is not dramatically modified during the sliding events [18].

By indicating with $\lambda=(\boldsymbol{q}, j)$ a phonon mode with wave vector $\boldsymbol{q}$ and band index $j$, the decrease of the population of the sliding mode $\lambda$ occurs at a transition rate $\mathcal{P}_{\lambda, \lambda^{\prime}}^{\lambda^{\prime \prime}}$ involving the $\lambda^{\prime}$ and $\lambda^{\prime \prime}$ dissipative modes, and is proportional to the square of the interaction strength $\Phi_{\lambda \lambda^{\prime} \lambda^{\prime \prime}}$ [37]:

$$
\mathcal{P}_{\lambda, \lambda^{\prime}}^{\lambda^{\prime \prime}} \propto n n^{\prime}\left(n^{\prime \prime}+1\right)\left|\Phi_{\lambda \lambda^{\prime} \lambda^{\prime \prime}}\right|^{2},
$$

where $n, n^{\prime}$ and $n^{\prime \prime}$ are the phonon populations, while $\Phi_{\lambda \lambda^{\prime} \lambda^{\prime \prime}}$ is a characteristic of the system. The phonon populations depend on the environment, e.g., the presence of thermal bath and irradiation or external intervention manifesting as external forces, load, and controlled sliding velocity. Such external parameters depend on the user, who can employ them to dynamically control the dissipation channels. We are here interested instead in modifying the intrinsic properties of the system to inhibit or mitigate specific dissipative processes, irrespective of the environment conditions. In fact, irrespective of the $n, n^{\prime}$, and $n^{\prime \prime}$ populations, if $\left|\Phi_{\lambda \lambda^{\prime} \lambda^{\prime \prime}}\right|^{2}$ is null, likewise the scattering probability in Eq. (1) is null and no dissipation will occur. A fine-tuning of the interaction strength tensor $\Phi_{\lambda \lambda^{\prime} \lambda^{\prime \prime}}$ then allows to design tribological materials with controlled frictional response: as $\left|\Phi_{\lambda \lambda^{\prime} \lambda^{\prime \prime}}\right|^{2}$ becomes lower, the $\lambda+\lambda^{\prime}=$ $\lambda^{\prime \prime}$ scattering becomes less probable and the lifetime of the sliding phonon $\lambda$ increases. This implies that the magnitude of an external drift force needed to keep the sliding active is small when low values of $\left|\Phi_{\lambda \lambda^{\prime} \lambda^{\prime \prime}}\right|^{2}$ are realized.

The interaction strength tensor $\Phi_{\lambda \lambda^{\prime} \lambda^{\prime \prime}}$ is an intrinsic property of the material since it is determined by the atomic kinds and geometry forming the system which, in turn, determine the eigenvectors, eigenfrequencies, and interatomic force constants [36,37]:

$$
\begin{aligned}
\Phi_{\lambda \lambda^{\prime} \lambda^{\prime \prime}}= & \sqrt{\frac{\hbar^{3}}{8 N^{3}}} \frac{1}{\sqrt{\omega_{\lambda} \omega_{\lambda^{\prime}} \omega_{\lambda^{\prime \prime}}}} \sum_{k k^{\prime} k^{\prime \prime}} \frac{1}{\sqrt{m_{k} m_{k^{\prime}} m_{k^{\prime \prime}}}} \\
& \times \sum_{\alpha \beta \gamma} e_{\lambda}^{\alpha}\left(\boldsymbol{r}_{k}\right) e_{\lambda^{\prime}}^{\beta}\left(\boldsymbol{r}_{k^{\prime}}\right) e_{\lambda^{\prime \prime}}^{\gamma}\left(\boldsymbol{r}_{k^{\prime \prime}}\right) \\
& \times \sum_{l l^{\prime} l^{\prime \prime}} e^{i \boldsymbol{q} \cdot \boldsymbol{r}_{k l}} e^{i \boldsymbol{q}^{\prime} \cdot \boldsymbol{r}_{k^{\prime} l^{\prime}}} e^{i \boldsymbol{q}^{\prime \prime} \cdot \boldsymbol{r}_{k^{\prime \prime} l^{\prime \prime}}} \Phi_{\alpha \beta \gamma}\left(\boldsymbol{r}_{k l}, \boldsymbol{r}_{k^{\prime} l^{\prime}}, \boldsymbol{r}_{k^{\prime \prime} l^{\prime \prime}}\right),
\end{aligned}
$$

where $\hbar$ is the reduced Planck's constant, $N$ is the number of unit cells, $\omega_{\lambda}$ is the eigenfrequency of the mode $\lambda, m_{k}$ is the mass of the $k$ th atom, $\boldsymbol{r}_{k l}\left(\boldsymbol{r}_{k^{\prime} l^{\prime}}, \boldsymbol{r}_{k^{\prime \prime} l^{\prime \prime}}\right)$ is the position of the $k$ th

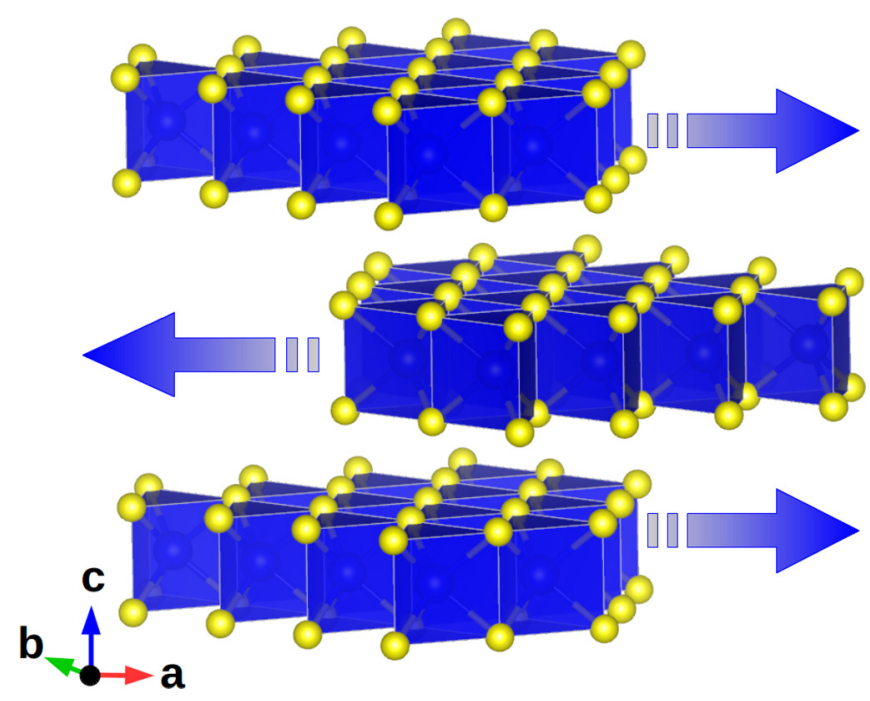

FIG. 2. Schematic example of one of the possible sliding modes in $M X-3 L$ systems: three adjacent $M X_{2}$ layers rigidly shifts along opposite directions according to the eigenvector $\boldsymbol{e}_{\Gamma, 6}$.

( $k^{\prime}$ th, $k^{\prime \prime}$ th) atom in the $l$ th $\left(l^{\prime}\right.$ th, $l^{\prime \prime}$ th) cell replica, $e_{\lambda}^{\alpha}\left(\boldsymbol{r}_{k}\right)$ is the $\alpha$ th Cartesian component of the eigenvector associated to the mode $\lambda$ and to the $k$ th atom, and $\Phi_{\alpha \beta \gamma}$ is the third-rank Cartesian tensor of the cubic anharmonic force constants. We already observed [38] that it is possible to turn off or on any $\lambda+\lambda^{\prime}=\lambda^{\prime \prime}$ scattering process by controlling the symmetries of the system; in fact, if $\Gamma^{\boldsymbol{e}_{\lambda}}, \Gamma^{\boldsymbol{e}_{\lambda^{\prime}}}$, and $\Gamma^{\boldsymbol{e}_{\lambda^{\prime \prime}}}$ are irreducible representations for which the eigenvectors $\boldsymbol{e}_{\lambda}, \boldsymbol{e}_{\lambda^{\prime}}, \boldsymbol{e}_{\lambda^{\prime \prime}}$ are, respectively, a basis, then

$$
\Phi_{\lambda \lambda^{\prime} \lambda^{\prime \prime}} \neq 0 \Rightarrow \Gamma^{\boldsymbol{e}_{\lambda}} \otimes \Gamma^{\boldsymbol{e}_{\lambda^{\prime}}} \otimes \Gamma^{\boldsymbol{e}_{\lambda^{\prime \prime}}} \subseteq A,
$$

which states that if the direct product among the irreducible representations contains the totally symmetric representation $A$, then the $\lambda+\lambda^{\prime}=\lambda^{\prime \prime}$ scattering is allowed. Indeed, this result is general and applies to any multiphonon scattering at any order of anharmonicity. In what follows, we show that an other way to control the value of $\Phi_{\lambda \lambda^{\prime} \lambda^{\prime \prime}}$ is to act on the subtle relation between the electronic density and the dynamic properties of the system.

In all the $M X-n L$ systems, the sliding modes with main contribution to relative layer shift have wave vector $\boldsymbol{q}=$ $(0,0,0) \equiv \Gamma$ and band indices which depend on $n$ [38] (see Fig. 2 as an example; a schematic representation of the sliding modes at different $n$ can be found in the Supporting Material of Ref. [38]). The number of sliding modes depends on the number of layers: the higher $n$, the higher the number of modes effectively contributing to the sliding, the higher the number of dissipative modes and corresponding dissipation channels. To track all the dissipative processes, we then consider the quantity $|\Phi|^{2}$, which we define as the sum of the squared modulus of all the $\Phi_{\lambda \lambda^{\prime} \lambda^{\prime \prime}}$ elements involving both $\lambda$ sliding and $\lambda^{\prime}, \lambda^{\prime \prime}$ dissipative modes. By controlling $|\Phi|^{2}$, we can then control the energy dissipation in tribological conditions at the nanoscale. A quick inspection of Eq. (2) shows that $\Phi_{\lambda \lambda^{\prime} \lambda^{\prime \prime}}$ can be decreased by simply choosing atoms with higher atomic masses $m_{k}$ via isotope substitution: however, this would cause a decrease of the eigenfrequencies $\omega_{\lambda}$, the 
two quantities being related by a relation of the kind $\omega \propto$ $1 / \sqrt{m}$. Instead, we will focus on the choice of the atomic type, which is the most adopted solution in practical applications.

The crucial quantity that determines $\Phi_{\lambda \lambda^{\prime} \lambda^{\prime \prime}}$ is the thirdorder tensor of the anharmonic force constants defined as

$$
\begin{aligned}
\Phi_{\alpha \beta \gamma}\left(\boldsymbol{r}_{k l}, \boldsymbol{r}_{k^{\prime} l^{\prime}}, \boldsymbol{r}_{k^{\prime \prime} l^{\prime \prime}}\right) & =\frac{\partial^{3} V}{\partial \boldsymbol{r}_{k l} \partial \boldsymbol{r}_{k^{\prime} l^{\prime}} \partial \boldsymbol{r}_{k^{\prime \prime} l^{\prime \prime}}} \\
& =\frac{\partial^{2}}{\partial \boldsymbol{r}_{k^{\prime} l^{\prime}} \partial \boldsymbol{r}_{k^{\prime \prime} l^{\prime \prime}}}\left(\frac{\partial V}{\boldsymbol{r}_{k l}}\right),
\end{aligned}
$$

where $V$ is the potential energy which is a function of the atomic positions. The term

$$
\frac{\partial V}{\boldsymbol{r}_{k l}}=-\boldsymbol{F}\left(\boldsymbol{r}_{k l}\right)
$$

is obtained by calculating the forces $\boldsymbol{F}\left(\boldsymbol{r}_{k l}\right)$, which depend on the kind of atoms and the topologic environment in which they are embedded. The accuracy with which such forces are calculated affects the estimation accuracy of the dissipative processes; $a b$ initio descriptions of the interatomic forces should then be preferred whenever computationally affordable, especially if subtle electronic effects must be taken into account. This also shows that the atomic type, the geometry, and the consequent electronic distribution, determine concurrently the final $\Phi_{\lambda \lambda^{\prime} \lambda^{\prime \prime}}$ values in a nontrivial way. Since it is not simple to map the behavior of $\Phi_{\lambda \lambda^{\prime} \lambda^{\prime \prime}}$ against the atomic kind, we need to identify proper collective descriptors to guide us through the complex interplay between the electronic structure and the dynamic features of the system. To this aim, we will investigate what are the relations among phononic states, electronic density, and phonon-phonon interactions.

\section{RESULTS AND DISCUSSION}

We begin our analysis by noticing that, at fixed chemical composition, no significant variation of the $a$ and $b$ lattice parameters is observed with the number of layers. Irrespective of the kind of $M$ and $X$ ions, $|\Phi|^{2}$ monotonically increases with increasing $n$ [Fig. 3(a)], in correspondence with the increase of the number of active dissipation channels. We then define $\bar{\omega}$ as the average frequency of the sliding modes, with the aim to capture how the eigenfrequencies determine the value of $|\Phi|^{2}$ at different number of layers. We observe that $\bar{\omega}$ is globally decreasing with $n$ but the trend is not monotonic [Fig. 3(b)]; indeed, at a fixed number of layers, higher $|\Phi|^{2}$ is realized at higher frequencies [Figs. 3(c) and 3(d)], against the intuitive trend suggested by Eq. (2), where the eigenfrequencies appear at the denominator of the expression of $\Phi_{\lambda \lambda^{\prime} \lambda^{\prime \prime}}$. Since by fixing $n$ we are making a comparison at a fixed topology, the $\boldsymbol{e}_{\lambda}$ vectors do not change significantly; the anharmonic force constants have then a key role in governing $\Phi_{\lambda \lambda^{\prime} \lambda^{\prime \prime}}$ since, in this case, they depend only on the atomic kinds and how these determine the electronic density. To get more insight on how to control $|\Phi|^{2}$, we then need to analyze in detail the subtleties of the electronic distribution. To this aim, we analyze the covalency [39] $\mathrm{C}_{M, X}$ of the $M-X$ bond, defined in terms of atomic contributions to the system wave function. The higher the $\mathrm{C}_{M, X}$ value, the larger the covalent character of the $M-X$ bond; equivalently, the lower the $\mathrm{C}_{M, X}$ value, the more ionic

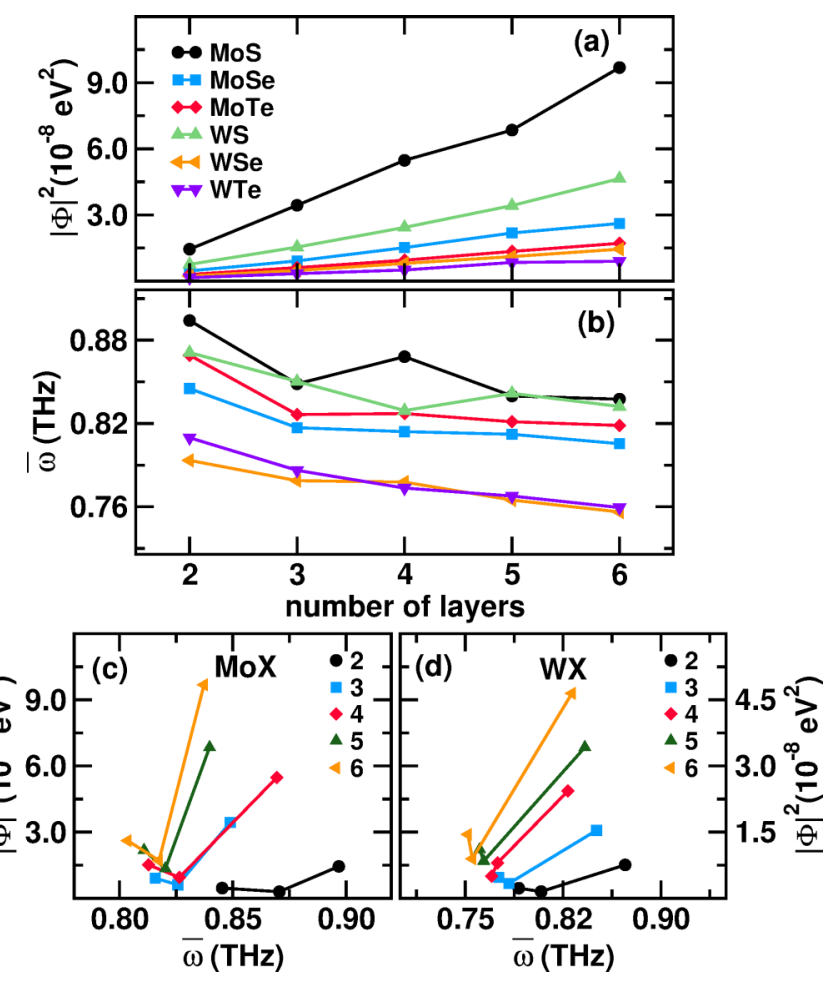

FIG. 3. (a) Sum of the square modulus of the interaction strengths involving both sliding and dissipative modes against the number of layers: the number of dissipation channels increases with $n$, producing an increase of the dissipated energy. (b) Average frequency of the sliding modes against the number of layers: at fixed composition the trend is not monotonic with $n$, showing the nontrivial relation between electronic and dynamic features of the systems; symbols are the same as in (a). (c),(d) $|\Phi|^{2}$ values of MoX- $n \mathrm{~L}$ and $\mathrm{WX}-n \mathrm{~L}$ systems against eigenfrequency average comprising both sliding and dissipative modes: higher dissipation may be realized at higher frequencies due to the competition between $\omega_{\lambda}$ and $\Phi_{\alpha \beta \gamma}$ values; the value of $n$ is indicated by the different symbols as shown in the legend. Lines are a guide for the eye.

the $M-X$ bond. We observe that the covalency can be considered constant with the number of layers [Fig. 4(a)]; the same result is obtained when we consider the difference among the atomic charges obtained either by integration of the atomprojected density of states or by performing a Bader analysis [40-43]. Irrespective of chemical composition and number of layers, we find that the more covalent the $M-X$ bond, the higher the $|\Phi|^{2}$ values hence the higher is the energy dissipation during layer sliding [Figs. 4(b) and 4(c)]. Concerning the dynamic aspect, at fixed $n$ highest $M-X$ bond covalency realizes highest average frequencies $\bar{\omega}$ (Fig. 5); however, as we already observed, an increase of the $M-X$ bond covalency produces an increase of the dissipation [Figs. 3(b) and 3(c)], against the fact that the eigenfrequencies $\omega_{\lambda}$ appear at the denominator of the expression of $\Phi_{\lambda \lambda^{\prime} \lambda^{\prime \prime}}$ [Eq. (2)]. This then confirms that the dominant contributions to the $\Phi_{\lambda \lambda^{\prime} \lambda^{\prime \prime}}$ values are represented by the interatomic force constants. Analysis of the electron localization functions $[44,45]$ does not show any strong evidence of charge redistribution along the $M-X$ bond at varying chemical composition [46]. This is the result of the subtle interplay between geometry, atomic kind, and 

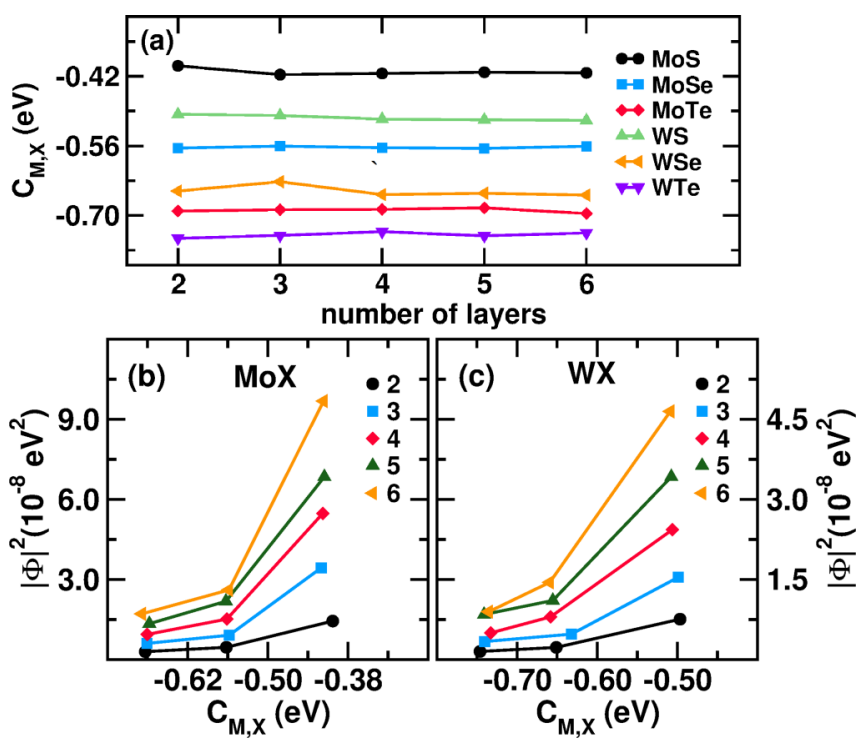

FIG. 4. (a) $M-X$ bond covalency against the number of layers: at fixed composition, covalency does not change noticeably with varying $n$. (b), (c) $|\Phi|^{2}$ values of MoX- $n \mathrm{~L}$ and WX- $n \mathrm{~L}$ systems against the $M-X$ bond covalency: irrespective of the chemical composition and the number of layers, more covalent bonds favor energy dissipation; the value of $n$ is indicated by the different symbols as shown in the legend. Lines are a guide for the eye.

electronic distribution, which can be uncovered by a deeper investigation.

To this aim, we consider the orbital polarization [47-49] of the $d$ and $p$ orbital projections of the $M$ and $X$ ions, respectively [50].

Orbital polarization $\mathcal{P}_{l_{1} m_{l 1}, l_{2} m_{l 2}}$ of the $\left|l_{1} m_{l 1}\right\rangle$ orbital relative to the $\left|l_{2} m_{l 2}\right\rangle$ orbital is defined as

$$
\mathcal{P}_{l_{1} m_{l 1}, l_{2} m_{l 2}}=\frac{n_{l_{1} m_{l 1}}-n_{l_{2} m_{l 2}}}{n_{l_{1} m_{l 1}}+n_{l_{2} m_{l 2}}},
$$

where $n_{l_{1} m_{l 1}}$ and $n_{l_{2} m_{l 2}}$ are the populations of $\left|l_{1} m_{l 1}\right\rangle$ and $\left|l_{2} m_{l 2}\right\rangle$, with orbital quantum number $l_{i}$ and magnetic quantum number $m_{l i}$, respectively. It measures the charge excess of the former orbital with respect to the latter: positive (negative) values indicate that $\left|l_{1} m_{l 1}\right\rangle$ orbital is more (less) populated than $\left|l_{2} m_{l 2}\right\rangle$ orbital. We observe that $\mathcal{P}_{p_{x}, p_{y}}, \mathcal{P}_{p_{x}, p_{z}}$ and $\mathcal{P}_{p_{y}, p_{z}}$

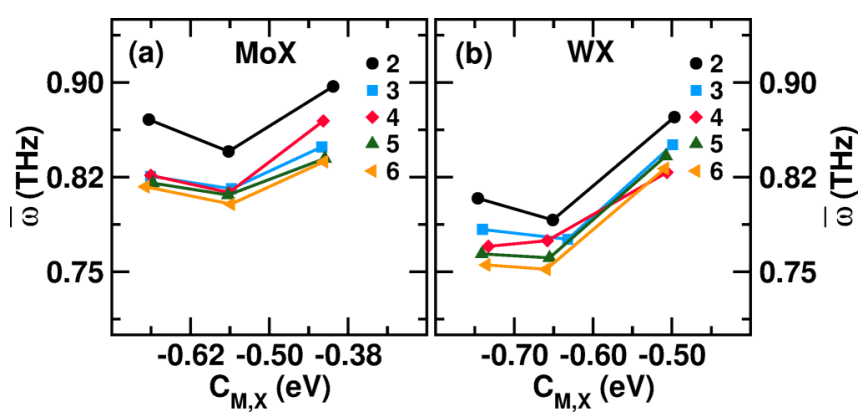

FIG. 5. Average frequency values of (a) MoX- $n \mathrm{~L}$ and (b) WX- $n \mathrm{~L}$ systems against the $M-X$ bond covalency: higher frequencies are realized at increased values of the bond covalency. Lines are a guide for the eye.

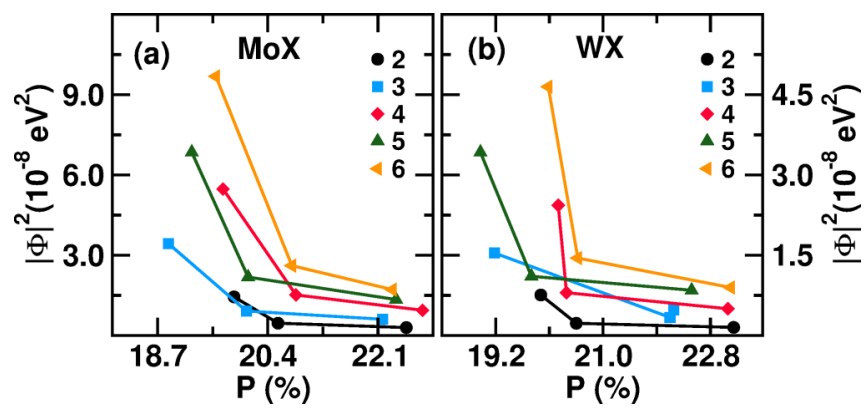

FIG. 6. $|\Phi|^{2}$ values of the (a) MoX- $n \mathrm{~L}$ and (b) WX- $n \mathrm{~L}$ systems as a function of the $\mathcal{P}_{t_{2 g}, e_{g}}$ orbital polarization. An excess of population in the $t_{2 g}$ orbitals reduce energy dissipation during layer sliding. Lines are a guide for the eye.

is almost constant with the number of layers. while the greatest variation is observed for $\mathcal{P}_{t_{2 g}, e_{g}}$ and $\mathcal{P}_{x^{2}-y^{2}, z^{2}}$. The symmetries of the system determine the relative occupation of the $d$ orbitals: $\mathcal{P}_{t_{2}, e_{g}}$ is higher (lower) for even (odd) number of layers; correspondingly, $\mathcal{P}_{x^{2}-y^{2}, z^{2}}$ has the opposite trend, while an exception is found for the WSe-3L system. A deeper analysis is needed to shed light on the origin of such behavior but for our purposes is not necessary and we will not investigate on it in this work. We already observed that the relative occupation of $t_{2 g}$ and $e_{g}$ orbitals plays an important role in the determination of the sliding dynamics in TMDs [26,49]; indeed, we find that an excess of electrons in the $t_{2 g}$ orbitals, reduces the $|\Phi|^{2}$ values hence the energy dissipation during layer sliding (Fig. 6). The delicate balance among the orbital populations determine the value of the covalency. At a fixed number of layers, lowest covalency is realized
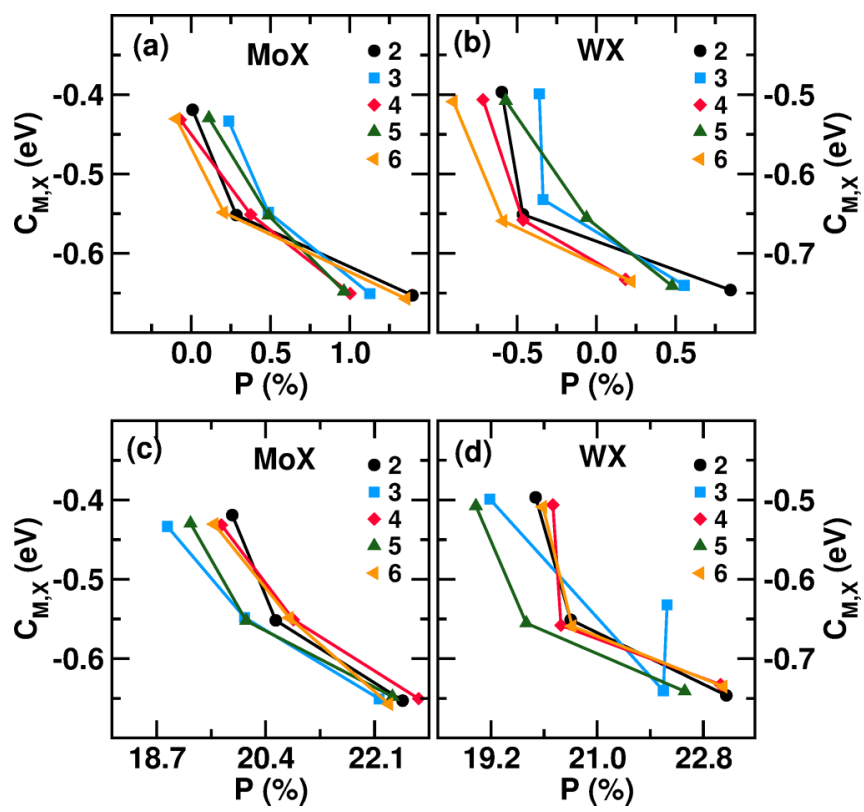

FIG. 7. Covalency of the $M X$ bond against the orbital polarization of MoX- $n \mathrm{~L}$ and WX- $n \mathrm{~L}$ systems: (a),(b) $X \mathcal{P}_{p_{x}, p_{y}}$, (c),(d) $M$ $\mathcal{P}_{t_{2 g}, e_{g}}$. Lowest covalency values are in general realized when the population unbalance between the considered atomic orbitals is the largest. Lines are a guide for the eye. 


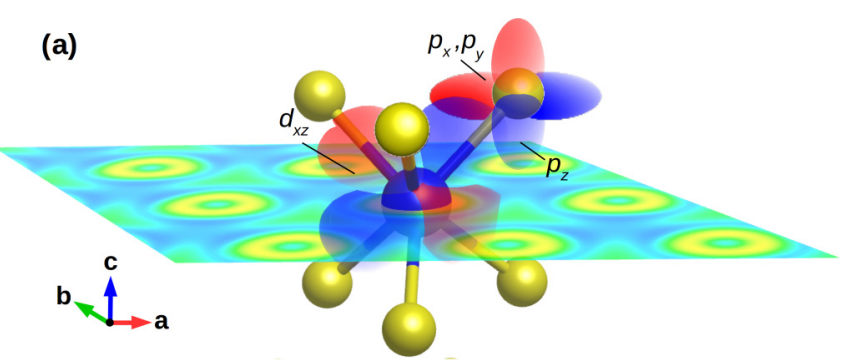

(b)

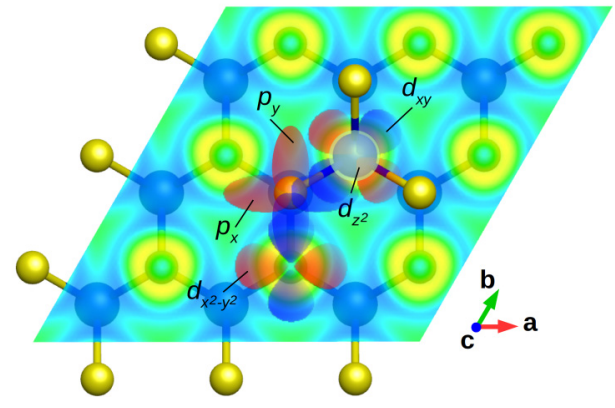

FIG. 8. Schematic of the electronic density projected on $t_{2 g}$ and $p$ orbitals, depicted as red and blue lobes and centered on the $M$ and $X$ atoms, respectively; the plane containing the $M$ cations is represented as a section of the electronic density, with RGB gradient showing density isovalues $($ red $=$ highest, blue $=$ lowest $)$. The $t_{2 g}$ and $p$ overlap is small, since the most of the $t_{2 g}$ orbitals extend into the region outside the $M-X$ bond axis; a charge shift towards the $t_{2 g}$ orbitals then makes the $M-X$ bond character more ionic. (a) Overlap among $d_{x z}$ (and $d_{y z}$ not shown for clarity), $p_{x}$ and $p_{y}$ is realized only by partial interpenetration of the orbital boundaries; (b) the same holds for the $d_{x y}$ and $p$ orbitals. The $d_{x^{2}-y^{2}}$ and $p_{y}$ overlap is more effective and an increase of their population makes the bond character more covalent. The $d_{z^{2}}$ orbital does not participate to the bond formation.

when the $p_{x}$ orbital results to be slightly more populated with respect to the $p_{y}$ orbital [Figs. 7(a) and 7(b)]; at the same time, low covalency values are in general realized when the population unbalance of the $t_{2 g}$ and $e_{g}$ orbitals is the largest possible [Figs. 7(c) and 7(d)]. High values of $\mathcal{P}_{p_{x}, p_{y}}$ and $\mathcal{P}_{t_{2},}, e_{g}$ hence determine the formation of more ionic $M-X$ bonds (Fig. 8) which lower the energy dissipation during sliding, in agreement with what we observed above [Figs. 4(b), 4(c), and 6].

We now need a descriptor which is able to parametrize the atomic types and their contribution to the sliding dynamics of the system. To this aim, we will use the cophonicity metric [16] defined in terms of atom-projected phonon density of states. We evaluate the cophonicity $\mathrm{C}_{p h}(M-X)$ of the $M-X$ pair in the frequency range $[0,1] \mathrm{THz}$, which corresponds to the eigenvectors with the highest contribution to the layer sliding. We observe that, irrespective of the chemical composition, cophonicity does not change significatively with the number of layers [Fig. 9(a)]. Positive $\mathrm{C}_{p h}(M-X)$ values indicate that $M$ and $X$ ions contribute more to higher- and lower-frequency displacements, respectively; this means that $M$ cations move faster than $X$ anions when forming the global layer sliding motion, while the opposite holds for negative $\mathrm{C}_{p h}(M-X)$ values. On the other hand, cophonicity close to zero corresponds to atomic displacements in which both $M$ and $X$ species move
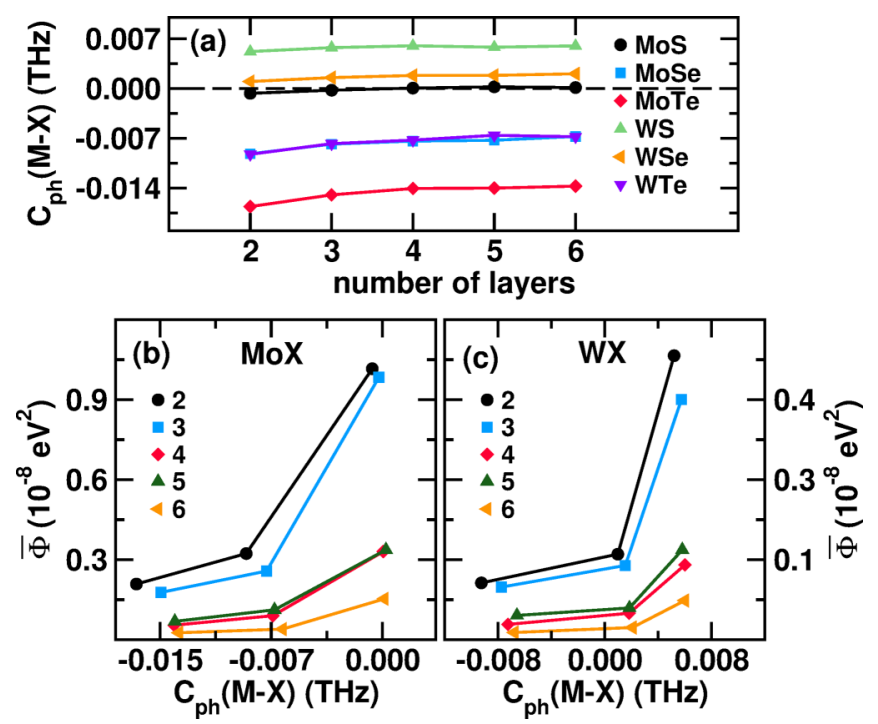

FIG. 9. (a) Cophonicity of the $M-X$ pair as a function of the number of layers; no significant variation is observed at fixed composition by varying $n$. (b, c) $\left|\Phi_{\lambda \lambda^{\prime} \lambda^{\prime \prime}}\right|^{2}$ values of MoX- $n \mathrm{~L}$ and WX- $n \mathrm{~L}$ systems against the $M-X$ pair cophonicity; lowest cophonicity favors low-energy dissipation during layer sliding. Lines are a guide for the eye.

in average at the same speed. At a fixed number of layers, lowest cophonicity realises lowest $|\Phi|^{2}$ values at fixed cation type [Figs. 9(b) and 9(c)], suggesting that when anions displace faster than cations, the frictional force and the corresponding energy dissipation is lower. This dynamical effect is connected to the spatial extent and directionality of the electronic distribution. In fact, at fixed $n, \mathrm{C}_{p h}(M-X)$ is monotonically increasing with $\mathrm{C}_{M, X}$ [Figs. 10(a) and 10(b)]; at the same time, $\mathrm{C}_{p h}(M-X)$ decreases with $\mathcal{P}_{t_{2 g}, e_{g}}$ becoming negative, indicating that a charge transfer towards $t_{2 g}$ orbitals favors a faster motion of the $X$ ions with respect to the $M$ cations when forming the global sliding motion [Figs. 10(c) and 10(d)]. In other words, a higher $t_{2 g}$ population changes the character of the sliding motion from $M$-dominant to $X$-dominant contributions to the overall layer shift. In our previous works $[16,27,49]$ we already used cophonicity to parametrize the atomic type and relate it to local harmonic forces which might contribute to the frictional response; here we can instead recognize that cophonicity can also be used to tune the anharmonic interactions which produce energy dissipation due to friction, hence going beyond the harmonic description.

Since covalency, cophonicity and orbital polarization descriptors appear to be tied together by simple monotonic relations, they can be easily fine-tuned to act as a knob to control $|\Phi|^{2}$, that is to control the energy dissipation due to the friction response during layer sliding. For example, we observed [16] that a specific $\mathrm{Ti} \rightarrow$ Mo cation substitution in $\mathrm{MoS}_{2}$ bulk induces local distortions in the ion environment which, in turn, determine a larger ionic bond character and a lower cophonicity. This coupled behavior resulted into a lowering of the sliding frequencies, and suggested that the individuated $\mathrm{Ti}: \mathrm{MoS}_{2}$ TMD phase should exhibit low friction when tangential external forces try to generate layer sliding. By following a similar approach, it is possible to 


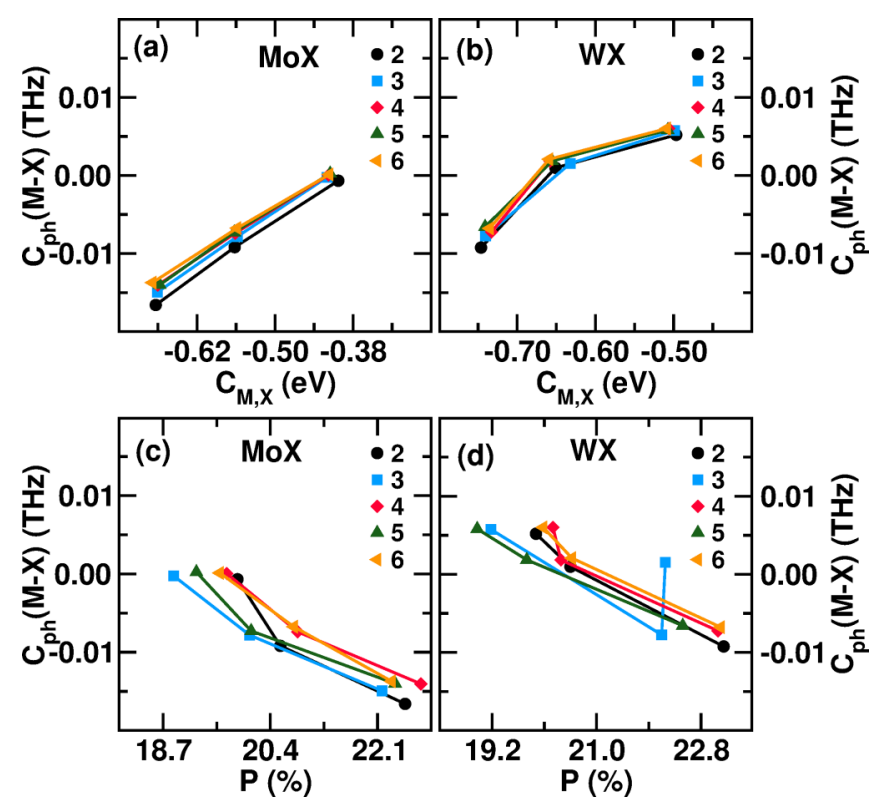

FIG. 10. (a),(b) Cophonicity of the $M-X$ pair of the MoX- $n \mathrm{~L}$ and WX- $n \mathrm{~L}$ systems as a function of the $M-X$ bond covalency: more covalent bond characters favor an increase of the $M-X$ cophonicity. (c),(d) Cophonicity of the $M-X$ pair as a function of the $\mathcal{P}_{t_{2 g}, e_{g}}$ orbital polarization: an excess of electrons in the $t_{2 g}$ orbitals favor a decrease of the cophonicity values. Lines are a guide for the eye.

use the relation among covalency, cophonicity, and orbital polarization to select the proper atomic species to design new tribological materials with controlled energy dissipation. We finally notice that calculation of cophonicity, covalency, and orbital polarization is faster than the evaluation of the phonon-phonon strength of interaction $\Phi_{\lambda \lambda^{\prime} \lambda^{\prime \prime}}$ since the latter requires the evaluation of atomic forces on hundreds of configurations each containing $\sim 200$ atoms. For this reason, the three descriptors can be used as a quick guide to select the proper atom to obtain low friction, or for fast high-throughput screening of potential low-dissipative layered materials.

\section{CONCLUSION}

We showed how nonequilibrium dissipative processes arising in tribological conditions can be controlled by fine-tuning the phonon-phonon interaction strengths, which are determined by the equilibrium configuration and atomic type of the system. In this way, it is possible to obtain information on the dynamic response without the need to run long and costly dynamic simulations. We applied our method to the study of layer sliding in transition metal dichalcogenides thin films. We recast the study of the frictional response in terms of sliding and dissipative phonons and observed that it is possible to tune the energy dissipation due to friction by fine tuning the sliding-dissipative phonon coupling. We found that an excess of charge in the $t_{2 g}$ orbitals of the $M$ cation favors the formation of more ionic $M-X$ bonds and reduces the energy dissipation; dynamically, the cophonicity descriptor shows that the lowered dissipation is obtained thanks to a faster motion of the $X$ atoms with respect to the $M$ cations. Cophonicity, covalency, and $\mathcal{P}_{t_{2 g}, e_{g}}$ orbital polarization are tied together by simple monotonic relations: the higher the $t_{2 g}$ orbital population, the more ionic the bond, the lower the cophonicity and the energy dissipation during sliding.

The combined use of electrostructural descriptors and phonon-based picture of the dissipative processes allows then to identify what are the relevant physical quantities to control the friction effect at the nanoscale. Such descriptors can also be used as parameters in high-throughput calculations or machine learning engines to screen large databases of compounds where anharmonic interactions are relevant. Finally, the presented approach is general and can be applied to the design of new tribological materials with targeted frictional response, irrespective of the chemistry and atomic topology.

\section{ACKNOWLEDGMENTS}

This work has been done with the support of the Czech Science Foundation (project No. 17-24164Y), and by the project "Novel nanostructures for engineering applications" No. CZ.02.1.01/0.0/0.0/16_026/0008396. This work was supported by The Ministry of Education, Youth and Sports from the Large Infrastructures for Research, Experimental Development and Innovations project "IT4Innovations National Supercomputing Center-LM2015070". The use of VESTA software [51,52] is also acknowledged.
[1] E. Gnecco and E. Meyer, Fundamentals of Friction and Wear on the Nanoscale, 2nd ed., NanoScience and Technology (Springer International Publishing, Switzerland, 2015), p. 704.

[2] A. Vakis, V. Yastrebov, J. Scheibert, L. Nicola, D. Dini, C. Minfray, A. Almqvist, M. Paggi, S. Lee, G. Limbert, J. Molinari, G. Anciaux, R. Aghababaei, S. E. Restrepo, A. Papangelo, A. Cammarata, P. Nicolini, C. Putignano, G. Carbone, S. Stupkiewicz, J. Lengiewicz, G. Costagliola, F. Bosia, R. Guarino, N. Pugno, M. Müser, and M. Ciavarella, Tribol. Int. 125, 169 (2018).

[3] A. Vanossi, N. Manini, M. Urbakh, S. Zapperi, and E. Tosatti, Rev. Mod. Phys. 85, 529 (2013).
[4] T. A. Sharp, L. Pastewka, and M. O. Robbins, Phys. Rev. B 93, 121402(R) (2016).

[5] D. Dietzel, J. Brndiar, I. Štich, and A. Schirmeisen, ACS Nano 11, 7642 (2017).

[6] A. Vanossi, D. Dietzel, A. Schirmeisen, E. Meyer, R. Pawlak, T. Glatzel, M. Kisiel, S. Kawai, and N. Manini, Beilstein J. Nanotechnol. 9, 1995 (2018).

[7] M. Igarashi, A. Natori, and J. Nakamura, Phys. Rev. B 78, 165427 (2008).

[8] W. K. Kim and M. L. Falk, Phys. Rev. B 80, 235428 (2009).

[9] P. Steiner, R. Roth, E. Gnecco, A. Baratoff, S. Maier, T. Glatzel, and E. Meyer, Phys. Rev. B 79, 045414 (2009). 
[10] D. Perez, Y. Dong, A. Martini, and A. F. Voter, Phys. Rev. B 81, 245415 (2010).

[11] E. Cihan, S. Ipek, E. Durgun, and M. Z. Baykara, Nat. Commun. 7, 12055 (2016).

[12] M. Z. Baykara, M. R. Vazirisereshk, and A. Martini, Appl. Phys. Rev. 5, 041102 (2018).

[13] Z. Wei, Z. Duan, Y. Kan, Y. Zhang, and Y. Chen, J. Appl. Phys. 127, 015105 (2020).

[14] S. R. S. de Mello, M. E. H. M. da Costa, C. M. Menezes, C. D. Boeira, F. L. Freire, Jr., F. Alvarez, and C. A. Figueroa, Sci. Rep. 7, 3242 (2017).

[15] E. Panizon, G. E. Santoro, E. Tosatti, G. Riva, and N. Manini, Phys. Rev. B 97, 104104 (2018).

[16] A. Cammarata and T. Polcar, Inorg. Chem. 54, 5739 (2015).

[17] A. Cammarata and T. Polcar, Phys. Rev. B 96, 085406 (2017).

[18] A. Cammarata, P. Nicolini, K. Simonovic, E. Ukraintsev, and T. Polcar, Phys. Rev. B 99, 094309 (2019).

[19] M. Chhowalla, H. S. Shin, G. Eda, L.-J. Li, K. P. Loh, and H. Zhang, Nat. Chem. 5, 263 (2013).

[20] H. Tian, J. Tice, R. Fei, V. Tran, X. Yan, L. Yang, and H. Wang, Nano Today 11, 763 (2016).

[21] Y. Liu, N. O. Weiss, X. Duan, H.-C. Cheng, Y. Huang, and X. Duan, Nat. Rev. Mater. 1, 16042 (2016).

[22] J. P. Perdew, K. Burke, and M. Ernzerhof, Phys. Rev. Lett. 77, 3865 (1996).

[23] G. Kresse and J. Furthmüller, Comp. Mater. Sci. 6, 15 (1996).

[24] G. Kresse and D. Joubert, Phys. Rev. B 59, 1758 (1999).

[25] S. Grimme, J. Comput. Chem. 27, 1787 (2006).

[26] A. Cammarata and T. Polcar, RSC Adv. 5, 106809 (2015).

[27] A. Cammarata and T. Polcar, Phys. Chem. Chem. Phys. 18, 4807 (2016).

[28] B. Schönfeld, J. J. Huang, and S. C. Moss, Acta Crystallogr. B 39, 404 (1983).

[29] V. Kalikhman, Inorg. Mater. 19, 957 (1983).

[30] L. Brixner, J. Inorg. Nucl. Chem. 24, 257 (1962).

[31] W. Schutte, J. D. Boer, and F. Jellinek, J. Solid State Chem. 70, 207 (1987).

[32] V. L. Kalikhman, Neorg. Mater. 19, 1060 (1983).
[33] A. A. Yanaki and V. A. Obolonchik, Inorg. Mater. 9, 1855 (1973).

[34] A. Togo, F. Oba, and I. Tanaka, Phys. Rev. B 78, 134106 (2008).

[35] A. Togo, L. Chaput, and I. Tanaka, Phys. Rev. B 91, 094306 (2015).

[36] D. M. Wallace, Thermodynamics of Crystals (John Wiley \& Sons., New York, 1972).

[37] J. M. Ziman, Electrons and Phonons: The Theory of Transport Phenomena in Solids (Oxford University Press, New York, 2001).

[38] A. Cammarata, RSC Adv. 9, 37491 (2019).

[39] A. Cammarata and J. M. Rondinelli, J. Chem. Phys. 141, 114704 (2014).

[40] W. Tang, E. Sanville, and G. Henkelman, J. Phys.: Condens. Matter 21, 084204 (2009).

[41] E. Sanville, S. D. Kenny, R. Smith, and G. Henkelman, J. Comput. Chem. 28, 899 (2007).

[42] G. Henkelman, A. Arnaldsson, and H. Jónsson, Comput. Mater. Sci. 36, 354 (2006).

[43] M. Yu and D. Trinkle, J. Chem. Phys. 134, 064111 (2011).

[44] A. D. Becke and K. E. Edgecombe, J. Chem. Phys. 92, 5397 (1990).

[45] A. Savin, O. Jepsen, J. Flad, O. K. Andersen, H. Preuss, and H. G. von Schnering, Angew. Chem., Int. Ed. Engl. 31, 187 (1992).

[46] See Supplemental Material at http://link.aps.org/supplemental/ 10.1103/PhysRevB.102.085409 for more information on the section "Electron Localization Function Analysis".

[47] A. Cammarata and J. M. Rondinelli, Phys. Rev. B 87, 155135 (2013).

[48] M. J. Han, C. A. Marianetti, and A. J. Millis, Phys. Rev. B 82, 134408 (2010).

[49] A. Cammarata and T. Polcar, Nanoscale 9, 11488 (2017).

[50] See Supplemental Material at http://link.aps.org/supplemental/ 10.1103/PhysRevB.102.085409 for more information on the section "Orbital Polarization Analysis".

[51] K. Momma and F. Izumi, J. Appl. Cryst. 41, 653 (2008).

[52] K. Momma and F. Izumi, J. Appl. Crystallogr. 44, 1272 (2011). 